\title{
Replacement of event-generated memories of nonreinforcement with signal-generated memories of reinforcement during partial reinforcement training: Effect on resistance to extinction
}

\author{
STEVEN J. HAGGBLOOM, LANEEL LOVELACE, VICKIE R. BREWER, \\ SHELIA M. LEVINS, and JAMES D. OWENS \\ Arkansas State University, State University, Arkansas
}

\begin{abstract}
Event-generated memory refers to the memory of a reinforcement (R) or nonreinforcement (N) event from an immediately preceding trial; signal-generated memory refers to the memory of a temporally remote $\mathrm{R}$ or $\mathrm{N}$, retrieval of which is generated by presentation of a signal with which the memory is associated (Haggbloom, 1988). In each of three experiments, Group Signal-R received runway discrimination training in Phase 1 to establish a stimulus as a signal for $R$, and partial reinforcement training in Phase 2. An extinction test measured learning about the memory of nonreward $\left(\mathrm{S}^{N}\right)$-learning that occurs when $\mathrm{S}^{N}$ is retrieved on $\mathrm{R}$ trials that follow $\mathrm{N}$ trials. In Group Signal-R, those R trials were accompanied by the signal for $\mathrm{R}$, a treatment we hypothesized would generate retrieval of the memory of reinforcement $\left(\mathrm{S}^{\mathrm{R}}\right)$ so that signal-generated $S^{R}$ would replace event-generated $S^{N}$ as the operative memory, thereby eliminating the increased resistance to extinction normally produced by PRF training. In each experiment, Group Signal-R was less resistant to extinction than was a control group conditioned to respond to event-generated $\mathrm{S}^{\mathrm{N}}$. Extinction was as rapid in Group Signal-R as it was in a consistent reinforcement control group (Experiment 1) and in a group given intertrial reinforcements to interfere with learning about $\mathrm{S}^{\mathrm{N}}$ (Experiment 3). Experiment 2 tested two alternative interpretations of the failure to learn about $S^{N}$ in Group Signal-R. Those alternatives were found to be less viable than the hypothesis that the signal for $\mathrm{R}$ actively recruited retrieval of a competing memory.
\end{abstract}

The rat has a highly developed capacity to store and retrieve memories of goal events; for example, reinforcement $(\mathrm{R})$, nonreinforcement $(\mathrm{N})$, and those memories (represented here as $S^{R}$ and $S^{N}$, respectively) constitute a powerful source of stimulus control over instrumental responding (Capaldi, 1966; Haggbloom, 1979, 1981). Haggbloom (1988) distinguished between two sources of goal-related memories: those generated by events and those generated by signals. Event-generated memories are those stored in response to an $\mathrm{R}$ or $\mathrm{N}$ event on one trial and recalled on the very next trial. Signal-generated memories are representations of $\mathrm{R}$ or $\mathrm{N}$ from temporally remote trials-representations stored in long-term memory-that are activated by presentation of a signal with which the memory is associated.

The very substantial behavioral control exercised by event-generated memories has been demonstrated in partial reinforcement experiments (Capaldi, 1966, 1971), dis-

This work was supported in part by a Faculty Research Grant from Arkansas State University. S. J. Haggbloom presented Experiments 1 and 2 at the November 1988 meeting of the Psychonomic Society in Chicago. L. Lovelace is now at Memphis State University. V. R. Brewer is now at the University of Houston. Requests for reprints should be addressed to Steven J. Haggbloom, Department of Psychology, Arkansas State University, P.O. Box 2127, State University, AR 72467. crimination learning experiments (e.g., Capaldi, Berg, \& Morris, 1975; Haggbloom, 1979, 1981; Haggbloom \& Tillman, 1980), and recent serial learning investigations (e.g., Capaldi \& Miller, 1988; Capaldi \& Verry, 1981; Haggbloom, 1985).

Haggbloom (1988) reported that the stimulus properties of signal-generated $S^{N}$, and its capacity to regulate extinction behavior, and event-generated $S^{N}$ were essentially the same. In those experiments, rats were trained in Phase 1 on a feature-negative discrimination problem in a runway in which the presence of a stimulus signaled $\mathrm{N}$ and its absence signaled $R$. To generate retrieval of $S^{N}$, that stimulus was then presented on selected trials during Phase 2, which consisted of consistent reinforcement (CRF) training. Groups trained in this way were labeled Signal-N groups, because it was hypothesized that during Phase 2 they would learn to respond to signalgenerated $S^{N}$.

Extinction trials were administered after Phase 2 to measure signal-generated retrieval and learning about $S^{\mathrm{N}}$. All extinction trials are nonreinforced, and thus $S^{N}$ is retrieved on each trial. If the signal presentation successfully generated retrieval of $S^{N}$ on $R$ trials in Phase $2, S^{N}$ would become a signal for reinforcement and Signal-N subjects would be expected to run faster in extinction than would the CRF and the nonsignaled control subjects. In each of 
five experiments, Signal-N subjects were substantially more resistant to extinction than were the CRF subjects, an effect named the signal-generated partial-reinforcement extinction effect (PREE). The signal-generated PREE was found to be as large as the PREE produced by conventional partial reinforcement (PRF) training, in which subjects learn to respond to event-generated $S^{\mathrm{N}}$.

In the three experiments reported here, we further investigated the acquisition of behavioral control by signalgenerated memory and compared it with the control exercised by event-generated memory. Specifically, we sought to determine whether retrieval and consequent learning about signal-generated $S^{\mathbf{R}}$ could be manipulated in a manner analogous to that already accomplished with regard to $S^{N}$. In the experiments reported by Haggbloom (1988), signal-generated $S^{N}$ replaced event-generated $S^{R}$ as the operative memory on selected trials, resulting in learning about $S^{N}$ that otherwise would not have occurred. That is, signal-N subjects were conditioned to respond to $S^{N}$, and thus were more resistant to extinction than were CRF and nonsignaled control groups.

In each of the three experiments reported here, we altered the procedures used by Haggbloom (1988) so that, in Signal-R groups, signal-generated $S^{\mathbb{R}}$ replaced eventgenerated $S^{N}$ as the operative memory on trials selected so as to preclude learning about event-generated $S^{N}$ that otherwise would have occurred. We predicted decreased resistance to extinction in Signal-R groups relative to that in nonsignaled control groups conditioned to respond to event-generated $S^{N}$ (Group Event- $N$ in each experiment). We further predicted a level of extinction-responding in Signal-R groups comparable to that in groups conditioned to respond only to event-generated $S^{R}$ as a consequence of CRF training (Group CRF in Experiment 1) or intertrial reinforcements (Group ITR in Experiment 3). In Experiment 2 , we investigated the possibility that reduced resistance to extinction in Signal-R groups occurs not because signal-generated $S^{R}$ replaces event-generated $S^{N}$ as the operative memory, but because our experimental procedures result in $R$ and $N$ trials for Group Signal-R that $\alpha c-$ cur in different stimulus contexts. That arrangement could interfere with stimulus control by $\mathbf{S}^{\mathbf{N}}$ independently of any putative replacement of $S^{N}$ with signal-generated $S^{R}$.

In addition to extending our knowledge about signalgenerated memory processes, the present experiments help to rule out alternatives to the signal-generated-memory interpretation of the results reported by Haggbloom (1988). Those alternatives, in terms of frustration theory (Amsel, 1958) and the possibility of enhanced conditioning due to surprise or a low expectancy of reinforcement resulting from the presentation of a signal for $\mathrm{N}$ on $\mathrm{R}$ trials, will be described more fully and in relation to the present experiments in the General Discussion section of this article.

\section{EXPERIMENT 1}

The purpose of Experiment 1 was to determine whether signal-generated $S^{\mathbf{R}}$ could be manipulated in such a way as to prevent learning about event-generated $S^{N}$ that would otherwise occur. Three groups of rats, identified as Groups Signal-R, Event-N, and CRF, were initially trained on a feature-positive discrimination problem, on which $\mathbf{R}$ trials were signaled by a tactile stimulus and $\mathrm{N}$ trials were unsignaled, to establish the tactile stimulus as a signal for reinforcement. In Phase 2, Groups Signal-R and Event- $\mathrm{N}$ both received partial reinforcement training, and Group CRF received consistent reinforcement training. Group Signal-R received the signal to generate retrieval of $S^{R}$ on $R$ trials that followed $N$ trials, trials on which event-generated $S^{N}$ would otherwise have been operative. Thus, subjects in Group Signal-R should learn about $S^{R}$ and be prevented from learning about $S^{N}$. Group Event-N, like a conventional PRF group, was conditioned to respond to event-generated $S^{N}$ on $R$ trials that followed $\mathrm{N}$ trials, and experienced the signal for $\mathrm{R}$ on $\mathrm{R}$ trials that did not follow $\mathrm{N}$ trials. We predicted that the subjects in Group Event-N would be more resistant to extinction than the subjects in Group CRF, which would be considered a partial reinforcement effect, and that the subjects in Group Signal-R would respond essentially like those in Group CRF.

\section{Method}

\section{Subjects}

The subjects were 18 male rats selected randomly from our laboratory colony of Holtzman-derived stock. The rats were $90-100$ days old at the beginning of experimental training.

\section{Apparatus}

The apparatus consisted of a single straight alleyway that was $235 \mathrm{~cm}$ long, $10 \mathrm{~cm}$ wide, and $15 \mathrm{~cm}$ high. The alleyway was made of wood and had a hinged, hardware-cloth top. The initial $25 \mathrm{~cm}$ and final $25 \mathrm{~cm}$ of the runway constituted start- and goalboxes, respectively. The startbox and goalbox were separated from the rest of the runway by manually operated guillotine doors. Three clocks $(.01 \mathrm{sec})$ recorded start, run, and goal times over respective distances of $20 \mathrm{~cm}$, the next $150 \mathrm{~cm}$, and the next $30 \mathrm{~cm}$ from the startbox door. Opening the startbox door activated the first clock; photoelectric circuitry controlled its offset and the operation of the remaining two clocks. The goalbox contained an unpainted goal cup that we constructed by drilling a 6-cm-diam hole in a small block of wood. The goal cup was positioned against the end wall of the goalbox.

\section{Procedure}

The experiment was conducted in two replications; the procedures described below refer to a single replication, unless stated otherwise. The rats were housed in individual cages with access to water throughout the experiment. Two weeks prior to the start of training, they were placed on a food-deprivation regimen, in which they were fed $12 \mathrm{~g}$ of lab chow per day, minus the weight of food pellets they would receive during training, throughout the experiment. On Days 12-14 of deprivation, the rats were handled in squads of 3 for 3 min per squad. After being handled on those days, they were fed eight $.045-\mathrm{g}$ food pellets from a small dish in the home cage. Three rats were randomly assigned to each of three groups: Groups Signal-R, Event-N, and CRF. Combining the two replications yields a total of 6 subjects per group.

Phase 1. Phase 1 training began on Day 15 of deprivation. All three groups were given identical training on a feature-positive discrimination problem in which all $\mathrm{R}$ trials were signaled and all $\mathrm{N}$ 
trials were unsignaled. The signal consisted of a strip of wire mesh (tactile stimulus) that covered the startbox floor and the floor of the runway up to the entrance to the goalbox (see Haggbloom, 1988, Experiment 2).

All subjects received six trials per day for 12 days, divided into two daily three-trial sessions administered at least $15 \mathrm{~min}$ apart. Within each session, the intertrial interval (ITI) was approximately $3 \mathrm{~min}$. The reinforcement sequence was RRN in Session 1 and RNN in Session 2 on odd-numbered days, and RNN in Session 1 followed by RRN in Session 2 on even-numbered days.

Phase 2. In Phase 2, Groups Signal-R and Event-N received 5 days of training on the partial reinforcement schedules shown in Table 1. As indicated in the table, the signal for reinforcement occurred on R trials that followed $\mathbf{N}$ trials for Group Signal-R. The signal occurred on $\mathrm{R}$ trials that did not follow $\mathrm{N}$ trials for Group Event-N. Group CRF received unsignaled reinforcement on all Phase 2 trials. After Phase 2, each subject received 30 unsignaled extinction trials at the rate of three trials per day.

Trial administration. At the beginning of each trial, a rat was placed in the startbox and the startbox door was opened approximately $3 \mathrm{sec}$ later. In each of the three phases of training, the rats were run in squads containing 1 rat from each group. The order of running rats within a squad was randomly varied across days, but the order of successive squads was held constant. All trials were separated by a 3-min ITI except, as noted above, for the ITI between Trial 3 of Session 1 and Trial 1 of Session 2, which was at least $15 \mathrm{~min}$. Rats received eight $.045-\mathrm{g}$ food pellets on all $\mathrm{R}$ trials and were confined to the goalbox for $20 \mathrm{sec}$ on all $\mathrm{N}$ trials.

A maximum time of $60 \mathrm{sec}$ was allowed in each section of the runway. If $60 \mathrm{sec}$ was exceeded in any section, the additional time was added to the time score of the next section forward. If the rat did not enter the goalbox in $180 \mathrm{sec}$, it was placed in the goalbox.

\section{Results}

In each of the three experiments, all analyses were based on speeds in centimeters per second $(\mathrm{cm} / \mathrm{sec})$, and only total speeds are reported here. The criterion of significance was set at $p<.05$ for all statistical tests.

\section{Phase 1}

In Phase 1 (data not shown), each group learned to run faster on signaled $\mathrm{R}$ trials than on unsignaled $\mathrm{N}$ trials. The mean running speeds (in $\mathrm{cm} / \mathrm{sec}$ ) were $85.66,79.00$, and 74.33 on R trials on the last day of Phase 1 and 37.89 , 53.88, and 35.46 on $N$ trials for Groups Event-N, Signal$R$ and $C R F$, respectively.

A group (3) $\times$ replication $(2) \times$ discriminanda $(2) \times$ trial (3) analysis of variance (ANOVA) was applied to running speeds on the last day of Phase 1 . The ANOVA yielded a reliable group $x$ discriminanda interaction

Table 1

Phase 2 Reward Schedules

\begin{tabular}{|c|c|c|c|c|c|c|c|c|c|c|}
\hline Day & & & & & ched & & & & & \\
\hline 1 & $\mathbf{R}$ & & $\mathbf{N}$ & ITR & $\underline{\mathbf{R}}$ & $\mathbf{R}$ & & $\mathrm{N}$ & ITR & $\underline{\mathbf{R}}$ \\
\hline 2 & $\mathbf{N}$ & ITR & $\underline{\mathbf{R}}$ & & $\overline{\mathbf{R}}$ & $\mathbf{N}$ & ITR & $\underline{\mathbf{R}}$ & & $\overline{\mathbf{R}}$ \\
\hline 3 & $\mathbf{R}$ & & $\overline{\mathbf{R}}$ & & $\mathbf{N}$ & $\mathrm{N}$ & ITR & $\underline{\mathbf{R}}$ & & $\mathbf{R}$ \\
\hline 4 & $\mathbf{R}$ & & $\mathrm{N}$ & ITR & $\underline{\mathbf{R}}$ & $\mathbf{R}$ & & $\overline{\mathbf{N}}$ & ITR & $\underline{\mathbf{R}}$ \\
\hline 5 & $\mathrm{~N}$ & ITR & $\underline{\mathbf{R}}$ & & $\vec{R}$ & $\mathrm{~N}$ & ITR & $\underline{\mathbf{R}}$ & & $\frac{\pi}{R}$ \\
\hline
\end{tabular}

Note- $\mathbf{R}=$ reinforcement $\mathbf{N}=$ nonreinforcement; $\mathbf{R}=$ signaled trials for Group Signal-R in each experiment; ITR = location of intertrial reinforcements administered to Group ITR in Experiment 3

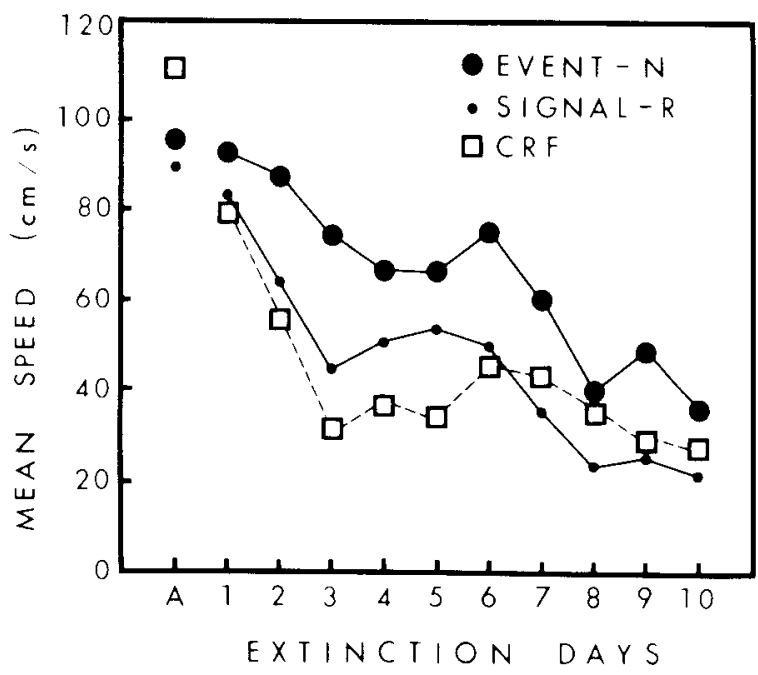

Figure 1. Mean speed on the last day of Phase 2 (A) and on each day of extinction for each of the three groups in Experiment 1.

$[F(2,12)=4.52]$. Simple effects of discriminanda at each group showed that all groups ran faster on signaled $\mathrm{R}$ trials than on unsignaled $\mathrm{N}$ trials [smallest $F(1,12)=21.91$ for Group Signal-R]. Simple effects of groups at discriminanda showed there were no differences among groups in $S+(F<1)$ or in $S-[F(2,12)=1.89]$. The group $\times$ discriminanda interaction reflects the somewhat smaller discrimination in Group Signal- $R$ relative to Groups Event-N and CRF.

\section{Phase 2}

There were no differences among the three groups in speed of running at the end of Phase 2. The mean running speed for each group on the last day of Phase 2 is presented as Point A in Figure 1. A group (3) $\times$ replication (2) $\times$ trial (6) ANOVA applied to those data showed there were no differences among groups $[F(2,12)=2.63]$ or between replications $(F<1)$; nor was there a group $X$ replication interaction $[F(2,12)=1.14]$.

\section{Extinction}

Figure 1 shows the mean running speed for each group on the last day of Phase 2 and on each day of extinction. As can be seen, Group Signal-R ran more slowly in extinction than Group Event- $\mathrm{N}$ and at about the same speed as Group CRF.

A group (3) $\times$ replication $(2) \times$ trial $(3) \times$ day (10) ANOVA applied to running speeds in extinction showed reliable differences among overall group mean extinction speeds $[F(2,12)=6.56]$, but no differences due to replications or the group $\times$ replication interaction $(F \mathrm{~s}<1)$. Subsequent planned comparisons showed that Groups Signal-R $(M=45.43)$ and CRF $(M=42.20)$ ran more slowly than Group Event $-\mathrm{N}\left[M=65.27 ; F_{\mathrm{s}}(1,12)=8.26\right.$ and 11.18] and that Groups Signal-R and CRF did not differ $(F<1)$. 


\section{Discussion}

As predicted, Experiment 1 demonstrated that a stimulus associated with reinforcement through Phase 1 discrimination training, and then presented on $R$ trials that followed $\mathrm{N}$ trials during a subsequent phase of PRF training, eliminated the increased resistance to extinction such PRF training otherwise would have produced. This outcome is consistent with our hypothesis that a signal associated with $S^{R}$ can be used to generate retrieval of that memory on selected learning trials. We activated $S^{R}$ in Group Signal-R on trials on which the operative memory otherwise would have been $S^{N}$, and thereby prevented learning about $S^{N}$ that otherwise would have occurred. The results obtained here as a consequence of manipulating signal-generated $S^{\mathbf{R}}$ complement results previously obtained by manipulating signal-generated $S^{\mathrm{N}}$ (Haggbloom, 1988; Haggbloom \& Brewer, 1989) and support a common explanation in terms of signal-generated memory processes for both sets of results.

\section{EXPERIMENT 2}

In Experiment 1, Group Signal-R failed to recall and learn to respond to the target memory, $S^{\mathbf{N}}$, during Phase 2 PRF training, as evidenced by the rapidity of extinction relative to Group Event-N. In Group Signal-R, but not in Group Event- $N, N$ and $R$ trials occurred in different stimulus contexts during Phase 2 . In Group Signal-R, $S^{\mathbf{N}}$ was produced and stored in a stimulus context that did not include the tactile cue established as a signal for $\mathbf{R}$ (we call this the wood-floor context). $\mathrm{N}$ trials were followed by signaled $R$ trials, that is, trials on which the tactile cue was present (we call this the tactile-cue context). According to our signal-generated memory hypothesis, the failure of Group Signal-R to learn about $S^{\mathbf{N}}$ occurred because the signal presentation (tactile-cue context) generated retrieval of $S^{\mathbb{R}}$, which then replaced $S^{N}$ as the operative memory. However, our procedures in Experiment 1 did not distinguish between that interpretation and two other possibilities.

First, Group Signal-R may have failed to learn about $S^{N}$ because the context change from wood floor to tactile cue simply failed to support retrieval of $S^{N}$ on $R$ trials (see, e.g., Spear, 1978). We will refer to this as the retrieval-failure hypothesis. The second possibility is that Group Signal-R may have retrieved $S^{N}$ on $R$ trials, but responding was then conditioned to a compound stimulus consisting of $S^{N}$ and the tactile cue. However, the stimulus compound present on unsignaled extinction trials consisted of $S^{N}$ and the wood floor. Thus, resistance to extinction would be greater in Group Event- $\mathrm{N}$, conditioned to respond to the same $S^{\mathrm{N}}$-plus-wood-floor compound, than in Group Signal-R. We call this the compound stimulus hypothesis.

In our second experiment, we investigated the possibility that reduced resistance to extinction in Group Signal-R might not be due to the replacement of $S^{N}$ by $S^{R}$ on signaled $R$ trials, but instead occurs for reasons given by the retrieval failure hypothesis or the compound stimulus hypothesis. In addition to Signal- $\mathrm{R}$ and Event- $\mathrm{N}$ groups trained as in Experiment 1, Experiment 2 included a group (labeled Group Context Change) that, like Group Signal-R, received $R$ trials in a context that was different from that experienced on $\mathrm{N}$ trials but that had not been established as a signal for $\mathbf{R}$. To the extent that the reduced resistance to extinction in Group Signal- $R$ is due to a retrieval failure or to conditioning to a compound stimulus different from that present on extinction trials, Group Context Change should also show reduced resistance to extinction.

\section{Method}

\section{Subjects}

The subjects were 12 male rats of the same description as in Experiment 1 . Four rats were randomly assigned to each of three groups.

\section{Apparatus}

The apparatus was the same as in Experiment 1.

\section{Procedure}

All procedures prior to the first day of training were the same as in Experiment 1 . In Phase 1, each group received 12 days of feature-positive discrimination training to establish the feature as a signal for $\mathbf{R}$. For one-half of the subjects in each group, the tactile cue used in Experiment 1 was established as a signal for $\mathbf{R}$. For the remaining subjects in each group, a brightness cue was established as a signal for $\mathrm{R}$. The brightness cue consisted of the placement of opaque covers over the hardware-cloth openings in the lids to the startbox and runway up to the goalbox. Thus, a darkened runway signaled $R$.

In Phase 2, each group received the same PRF schedules used in Experiment 1, as shown in Table 1. On signaled trials in Phase 2, each subject in Group Signal-R received the same stimulus (tactile cue or brightness cue) that had been established for that subject as a signal for $\mathrm{R}$ during Phase 1 training. Subjects in Group Context Change, on the other hand, received the opposite stimulus. Thus, if the tactile cue was established as the signal for $R$ for a subject in Phase 1, that subject received the brightness cue on signaled $R$ trials in Phase 2. In this way, Groups Signal-R and Context Change experienced the same degree of change between the storage context on $\mathbf{N}$ trials and the retrieval context on $\mathbf{R}$ trials. In the case of Group Signal-R, the retrieval context was associated with $S^{R}$, but in the case of Group Context Change it was not. Indeed, for Group Context Change, the retrieval context was at first novel. Group Event-N was trained as in Experiment 1, except that the tactile and brightness cues were not presented on any trials.

Following the 5 days of Phase 2 training, there were 10 days of extinction trials at the rate of three trials per day. All trial administration procedures were the same as in Experiment 1.

\section{Phase 1}

\section{Results}

In Phase 1 (data not shown), each group learned to run faster on signaled $\mathrm{R}$ trials than on unsignaled $\mathrm{N}$ trials. The mean running speeds (in $\mathrm{cm} / \mathrm{sec}$ ) were $79.18,83.62$, and 80.67 on $R$ trials on the last day of Phase 1 and 47.78, 42.89 , and 47.52 on $\mathrm{N}$ trials for Groups Signal-R, Event-N, and Context Change, respectively.

A group (3) $\times$ signal type (brightness or tactile [2]) $\times$ discriminanda $(2) \times$ trial $(3)$ analysis of variance 
(ANOVA) and subsequent planned comparisons applied to running speeds on the last day of Phase 1 yielded a reliable effect of discriminanda $[F(1,6)=20.32]$ and a reliable effect of signal type $[F(1,6)=22.47]$. The latter effect was obtained because the subjects given the tactile cue ran faster than did subjects given the brightness cue. There was no group $\times$ signal type interaction $(F<1)$, and nor did either of those variables interact with discriminanda [largest $F(1,6)=3.80$ for the signal type $\times$ discriminanda interaction]. Planned comparisons showed that all groups ran faster on signaled $R$ trials than on unsignaled $\mathrm{N}$ trials [smallest $F(1,6)=6.62$ for Group Signal-R].

\section{Phase 2}

There were no differences among the three groups in speed of running at the end of Phase 2. The mean running speed for each group on the last day of Phase 2 is presented as Point A in Figure 2. A group (3) $\times$ signal type (2) $\times$ trial (6) ANOVA applied to those data showed that there were no differences among groups $[F(2,6)=$ 1.33], no difference due to signal type $[F(1,6)=2.64]$, and no group $\times$ signal type interaction $(F<1)$.

\section{Extinction}

Figure 2 shows the mean running speed for each group on the last day of Phase 2 and on each day of extinction. As can be seen, Group Signal- $R$ ran more slowly in extinction than did Groups Event- $\mathrm{N}$ and Context Change, and the latter two groups responded similarly over the course of extinction.

A group (3) $\times$ signal type $(2) \times$ trial $(3) \times$ day $(10)$ ANOVA applied to running speeds in extinction yielded a significant main effect of groups $[F(2,6)=12.52]$, no differences due to signal type $[F(1,6)=1.17]$, and no

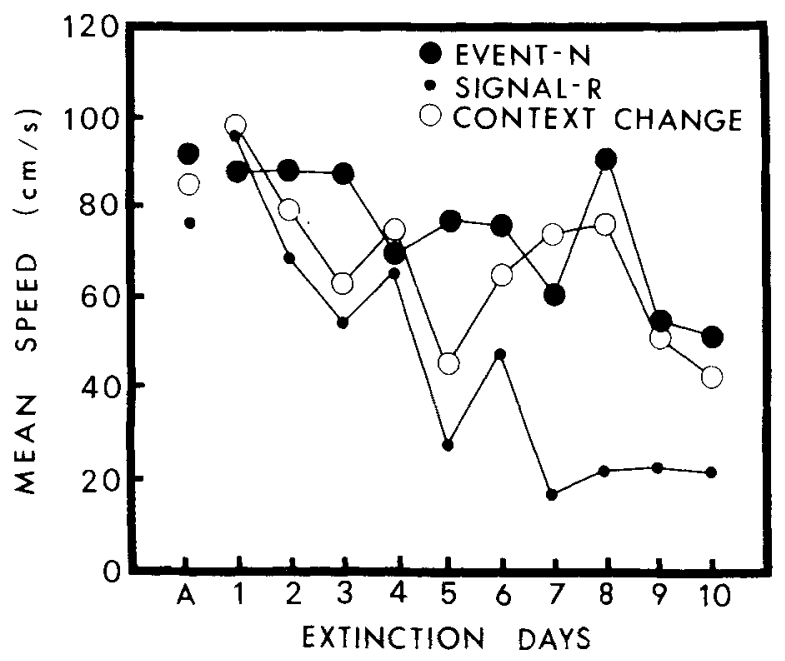

Figure 2. Mean speed on the last day of Phase 2 (A) and on each day of extinction for each of the three groups in Experiment 2. group $\times$ signal type interaction $(F<1)$. Planned comparisons performed on group mean extinction speeds showed that Groups Event-N $(M=74.62)$ and Context Change $(M=66.54)$ ran faster than Group Signal-R $(M=43.81)\left[F_{\mathrm{S}}(1,6)=23.29\right.$ and 12.67 , respectively $]$. Groups Event- $\mathrm{N}$ and Context Change did not differ in mean running speed during extinction $[F(1,6)=1.60]$.

\section{Discussion}

As in Experiment 1, resistance to extinction was decreased in Group Signal-R, relative to Group Event-N, by the presentation of a signal which had been associated with reinforcement on $\mathrm{R}$ trials that followed $\mathrm{N}$ trials during PRF training. The important finding in Experiment 2 was that Group Context Change did not show decreased resistance to extinction relative to Group Event-N.

Under our procedures in Experiments 1 and 2, $\mathrm{N}$ and $\mathbf{R}$ trials for Group Signal- $\mathbf{R}$ occurred in different stimulus contexts defined by the absence and presence, respectively, of the signal for R. In Experiment 2, Group Context Change experienced the same change in context accompanying $\mathbf{N}$ and $\mathbf{R}$ trials as did Group Signal- $R$, but the context present on $R$ trials had not been established as a signal for $\mathbf{R}$. For both groups, however, $S^{\mathbf{N}}$ stored in one context had to be recalled in the changed context in order to gain control over responding. The considerable resistance to extinction in Group Context Change suggests that $S^{N}$ was successfully retrieved on $R$ trials in the changed context in that group and exercised considerable control over extinction-responding in the wood-floor context. This outcome is inconsistent with both the retrievalfailure and compound-stimulus hypotheses. The results of Experiment 2 support the signal-generated-memory hypothesis, according to which the reduced resistance to extinction in Signal-R groups occurs because the signal generates retrieval of $S^{R}$ which then replaces $S^{N}$ as the operative memory. This precludes learning about $S^{N}$ and thus reduces resistance to extinction.

The finding here that a context change impaired recall of $S^{N}$ as the target memory only when the new context generated retrieval of a competing memory is consistent with the results of experiments on the effects of trial sequence on brightness-differential conditioning. In that situation, whether $S^{N}$ stored in the context of one runway is retrieved on the subsequent trial in the other runway depends on whether or not the two runway brightness cues have been established as discriminative stimuli (Haggbloom, 1980). When $\mathrm{N}$ trials in one runway are followed by $R$ trials in the other runway from the outset of training, and thus before the discrimination is learned, $S^{N}$ stored in one runway is retrieved in the other runway and gains considerable control over responding (Capaldi, Berg, \& Morris, 1975; Haggbloom, 1978, 1980; Haggbloom \& Tillman, 1980). On the other hand, if the discrimination is learned first, and only then are $\mathrm{N}$ trials in one runway followed by $\mathrm{R}$ trials in the other 
runway, $S^{N}$ gains very little control over behavior (Haggbloom, 1980). Presumably, this is because retrieval of $S^{N}$ is poor in a context that has come to signal reinforcement.

\section{EXPERIMENT 3}

The decreased resistance to extinction in Group Signal-R, relative to Group Event- $\mathbf{N}$, obtained in Experiments 1 and 2 is strongly reminiscent of the decreased resistance to extinction produced by intertrial reinforcements (ITRs) scheduled to follow $\mathrm{N}$ trials that precede $\mathrm{R}$ trials (e.g., Capaldi \& Spivey, 1963). In terms of the present analysis, ITRs occasion event-generated $S^{R}$ which then replaces event-generated $S^{N}$ as the operative memory. In Experiments 1 and 2, event-generated $S^{N}$ was replaced by signal-generated $S^{R}$. Both procedures effectively prevent learning about event-generated $S^{N}$ that otherwise would occur during PRF training. The purpose of Experiment 3 was to compare the capacity of signal-generated $S^{R}$ to replace $S^{N}$ as the operative memory with the capacity of event-generated $S^{R}$ occasioned by an ITR to do the same.

There were three groups in Experiment 3: Groups Signal-R, Event-N, and ITR. As in Experiment 1, each group received feature-positive discrimination training in Phase 1 to establish a tactile stimulus as a signal for reinforcement. In Phase 2, Groups Signal-R and Event-N were trained as in Experiment 1. Group ITR was trained like Group Event-N, except that all Phase 2 trials were unsignaled and ITRs were scheduled after all $\mathrm{N}$ trials that immediately preceded an R trial. According to our analysis, event-generated $S^{\mathbf{N}}$ will be replaced by signalgenerated $S^{R}$ in Group Signal-R and by event-generated $S^{R}$ in Group ITR; thus, learning about event-generated $S^{N}$ will be precluded in both groups. Consequently, we predict that Groups Signal-R and ITR will be equally resistant to extinction, and both groups will be less resistant to extinction than Group Event-N.

Method
Subjects
The subjects were 24 male rats of the same description as in Ex-
periments 1 and 2 . Four rats were randomly assigned to each of
three groups in both of two replications of the experiment.

\section{Apparatus \\ The apparatus was the same as in Experiment 1.}

\section{Procedure}

All procedures through the end of Phase 1 were identical to those in Experiment 1. In Phase 2, the schedules of PRF for all three groups were the same as those employed in Experiment 1. Group Signal-R received signaled trials on the same schedule as in Experiment 1 (see Table 1). Group ITR, as shown in Table 1, received rewarded placements in the goalbox (intertrial reinforcements, ITRs) before $\mathrm{R}$ trials that followed $\mathrm{N}$ trials, that is, immediately preceding trials in the same ordinal position as signaled trials for Group Signal-R. The ITR procedure involved removing the rat from the intertrial holding cage and placing it directly into the goal- box with its head directly over the goal cup, which was baited with eight $.045-\mathrm{g}$ food pellets. After the rat consumed the food pellets, it was placed directly into the startbox and administered the scheduled runway trial. There were 5 days of training in Phase 2, followed by 30 extinction trials administered at the rate of 3 trials per day. All other procedures were the same as in Experiment 1.

\section{Phase 1}

\section{Results}

In Phase 1 (data not shown), each group learned to run faster on signaled $\mathrm{R}$ trials than on unsignaled $\mathrm{N}$ trials. The mean running speeds (in $\mathrm{cm} / \mathrm{sec}$ ) were $85.70,83.37$, and 94.08 on $R$ trials on the last day of Phase 1 and $36.17,50.19$, and 49.91 on $N$ trials for Groups Signal-R, Event-N, and ITR, respectively.

A group (3) $\times$ replication $(2) \times$ discriminanda $(2) \times$ trial (3) analysis of variance (ANOVA) and subsequent planned comparisons applied to running speeds on the last day of Phase 1 yielded a reliable main effect of discriminanda $[F(1,18)=69.60]$, but no differences due to groups or replications, and no interactions between those variables and discriminanda [largest $F(2,18)=3.20$ for the group $\times$ discriminanda interaction]. Planned comparisons showed that all groups ran faster on signaled $\mathrm{R}$ trials than on unsignaled $\mathrm{N}$ trials [smallest $F(1,18)=13.82$ for Group Event-N].

\section{Phase 2}

There were no differences among the three groups in speed of running at the end of Phase 2. The mean running speed for each group on the last day of Phase 2 is presented as Point A in Figure 3. A group (3) $\times$ replication (2) $\times$ trial (6) ANOVA applied to those data showed that there were no differences among groups or between replications and no group $\times$ replication interaction (all $F_{\mathrm{s}}<1$ ).

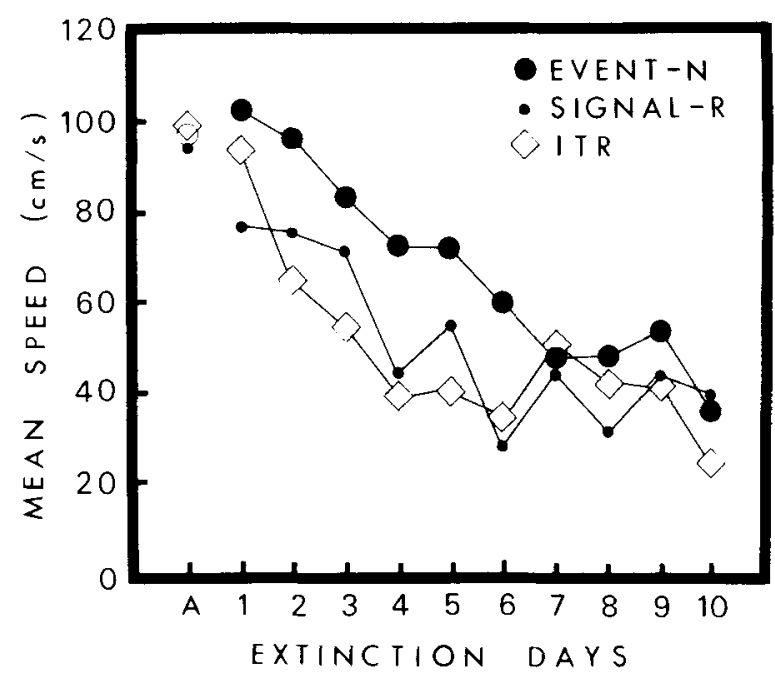

Figure 3. Mean speed on the last day of Phase 2 (A) and on each day of extinction for each of the three groups in Experiment 3. 


\section{Extinction}

Figure 3 shows the mean running speed for each group on the last day of Phase 2 and on each day of extinction. As can be seen, Group Signal-R ran more slowly in extinction than Group Event $-\mathrm{N}$ and at about the same speed as Group ITR.

A group $(3) \times$ replication $(2) \times \operatorname{trial}(3) \times$ day $(10)$ ANOVA applied to running speeds in extinction failed to yield a reliable groups effect $[F(2,18)=2.65]$ but did yield a reliable group $\times$ day interaction $[F(18,162)=$ 2.12]. Inspection of Figure 3 shows that differences among groups were most pronounced over Days 1-6, after which all three groups responded similarly. Therefore, the same ANOVA was applied to Days 1-6 and yielded a significant groups effect $[F(2,18)=4.92]$. There were no interactions with replications [largest $F(5,90)=1.01$ for the replication $x$ day interaction].

Planned comparisons performed on group mean extinction speeds over Days 1-6 showed that Group Event-N $(M=81.12)$ ran faster than Groups Signal-R $[M=58.79 ; F(1,18)=6.21]$ and ITR $[M=55.16$; $F(1,18)=8.39\}$. Groups Signal-R and ITR did not differ in mean running speed over Days 1-6 $(F<1)$.

\section{Discussion}

These results agree with those of Experiments 1 and 2 in showing that a stimulus associated with reinforcement, and presented on $\mathrm{R}$ trials that follow $\mathrm{N}$ trials during a separate phase of PRF training, eliminates the increased resistance to extinction such PRF training would otherwise produce. We have attributed this effect to the replacement of event-generated $S^{N}$ with signal-generated $S^{R}$ as the operative memory that precluded learning to respond to $\mathrm{S}^{\mathrm{N}}$.

The slower running in extinction in Group ITR than in Group Event-N agrees with the results of previous experiments (e.g., Capaldi \& Spivey, 1963) that have shown that ITRs experienced prior to $\mathrm{R}$ trials that follow $\mathrm{N}$ trials decrease resistance to extinction. According to our analysis, this effect of ITRs occurs because event-generated $S^{R}$ occasioned by the ITR replaces event-generated $S^{N}$ as the operative memory on trials on which subjects would otherwise have learned to respond to $S^{N}$.

The nearly identical extinction behavior of Groups Signal- $R$ and ITR in the present experiment suggests that signal-generated $S^{R}$ replaced event-generated $S^{N}$ and thus interfered with learning about $S^{N}$ as efficaciously as eventgenerated $S^{R}$ occasioned by an ITR. This result complements the finding that $S^{N}$ gains as much behavioral control in Signal-N groups as in groups given actual PRF training (Haggbloom, 1988, Experiments 4 and 5). Both findings indicate that the stimulus properties of signalgenerated memory are essentially the same as those of event-generated memory.

\section{GENERAL DISCUSSION}

Haggbloom (1988) reported that the presentation of a signal for $\mathrm{N}$ on selected $\mathrm{R}$ trials during CRF training in- creased resistance to extinction relative to CRF and nonsignaled control groups. Groups trained in this way were identified as Signal-N groups, and the increased resistance to extinction in those groups was called the signalgenerated partial-reinforcement extinction effect (PREE). The signal-generated PREE was attributed to the retrieval of $S^{N}$, generated by the signal presentation, on trials on which $\mathbf{S}^{\mathbf{R}}$ would otherwise have been the operative memory. Because retrieval of $S^{N}$ was generated on $R$ trials, responding was conditioned to $S^{N}$, thereby increasing resistance to extinction. The present finding that we can manipulate signal-generated $S^{\mathbf{R}}$ so that it replaces $S^{\mathbf{N}}$ as the operative memory supports a common interpretation of both sets of results in terms of signal-generated memory retrieval. Collectively, the two sets of results recommend against some alternative interpretations of the signalgenerated PREE.

Two interpretations of the signal-generated PREE are possible within the framework of frustration theory (Amsel, 1958, 1972). One of these, in terms of adaptation to the behaviorally disruptive effects of presenting a signal for $\mathrm{N}$ on $\mathrm{R}$ trials, has been addressed (Haggbloom \& Brewer, 1989). The second possibility is that the signal for $\mathrm{N}$ elicits anticipatory frustration to which subjects become counterconditioned during Phase $2 \mathrm{CRF}$ training. It is also possible that the signal-generated PREE is due to enhanced conditioning that occurs when there is a large discrepancy between expected and obtained reinforcement (see, e.g., Capaldi, 1974; Capaldi \& Haggbloom, 1974; Zanich \& Fowler, 1978). Presentation of a signal for N on $\mathrm{R}$ trials during Phase 2 CRF training would presumably lower expectancy of reinforcement.

The present experiments do not rule out an interpretation of the increased resistance to extinction previously obtained in Signal-N groups (Haggbloom, 1988) in terms of either frustration theory or enhanced conditioning. However, the finding here of decreased resistance to extinction in Signal-R groups is not amenable to an interpretation in terms of frustration theory, and decreased resistance to extinction is obviously not caused by enhanced conditioning. Thus, while frustration theory or the concept of enhanced conditioning might offer an explanation of the signal-generated PREE, the signal-generated memory processes described by Haggbloom (1988) provide a unitary explanation of the results reported here and of those reported previously (Haggbloom, 1988; Haggbloom \& Brewer, 1989).

\section{REFERENCES}

AMSEL, A (1958). The role of frustrative nonreward in noncontinuous reward situations. Psychological Bulletin, 55, 102-119.

AMSE L, A . (1972). Behavioral habituation, counterconditioning, and a general theory of persistence. In A. H. Black \& W. F. Prokasy (Eds.), Classical conditioning II: Current theory and research (pp. 409-426). New York: Appleton-Century-Crofts.

CAPALDI, E. J. (1966). Partial reinforcement: A hypothesis of sequential effects. Psychological Review, 73, 459-477.

CApaldi, E. I. (1971). Memory and learning: A sequential viewpoint. In W. K. Honig \& P. H. R. James (Eds.), Animal memory (pp. 111 154). New York: Academic Press. 
CAPALDI, E. J. (1974). Partial reinforcement either following or preceding consistent reward: A case of reinforcement level. Journal of Experimental Psychology, 102, 954-962.

CAPALDi, E. J., BerG, R. F., \& Morris, M. D. (1975). Stimulus control of responding in the early trials of differential conditioning. Leaming \& Motivation, 6, 217-229.

CAPAldi, E. J., \& HaGgBloom, S. J. (1974). Influence of reward magnitude on the initial nonreward effect. Bulletin of the Psychonomic Society, 3, 219-221.

CAPAldi, E. J., \& Miller, D. J. (1988). The rats' simultaneous anticipation of remote events and current events can be sustained by event memories alone. Animal Learning \& Behavior, 16, 1-7.

Capaldi, E. J., \& SPIVEY, J. (1963). The effect of goal box similarity on the aftereffect of nonreinforcement and resistance to extinction. Journal of Experimental Psychology, 66, 461-465.

CAPALDI, E. J., \& VERRY. D. R. (1981). Serial order anticipation learning in rats: Memory for multiple hedonic events and their order. Animal Learning \& Behavior, 9, 441-453.

HAGGBLOOM, S. J. (1978). Intertrial interval effects on internal stimulus control of behavior in brightness differential conditioning. Leaming \& Motivation, 9, 347-358.

HAGGBLoom, S. J. (1979). The differential reinforcement of rewardproduced and response-produced cues. Learning \& Motivation, 10 , 364-381.
HAGGBLOOM, S. J. (1980). Effects of training level and locus of N-R transitions on resistance to discrimination. Psychological Record, 30. 419-422.

HAGGBLOOM, S. J. (1981). Blocking in successive differential conditioning: Prior acquisition of control by internal cues blocks the acquisition of control by brightness. Leaming \& Motivation, 12, 485-508.

HAGGBLOOM, S. J. (1988). The signal-generated partial reinforcement extinction effect. Journal of Experimental Psychology: Animal Behavior Processes, 14, 83-95.

HaGgbloom, S. J., \& Brewer, V. R. (1989). Behavioral disruption versus signal-generated memory retrieval as determinants of the signalgenerated partial reinforcement extinction effect. Bulletin of the Psychonomic Society, 27, 99-102.

Haggbloom, S. J., \& Tillman, D. J. (1980). Sequential effects on discrimination reversal. Leaming \& Motivation, 11, 318-338.

SPEAR, N. E. (1978). Processing of Memories: Forgetting and retention. Hillsdale, NJ: Eribaum.

ZANich, M. L., \& FowleR, H. (1978). Transfer from Pavlovian appetitive to instrumental appetitive conditioning: Signaling versus discrepancy interpretations. Journal of Experimental Psychology: Animal Behavior Processes, 4, 37-49.

(Manuscript received November 23, 1989; revision accepted for publication April 14, 1990.) 